\title{
Laser Energy Scaling Law for the Yield of Neutrons Generated by Intense Femtosecond Laser-Cluster Interactions
}

\author{
Shuji SAKABE, Masaki HASHIDA, Shigeki TOKITA and Kazuto OTANI \\ Advanced Research Center for Beam Science, Institute for Chemical Research, Kyoto \\ University, Gokasho, Uji, Kyoto 611-001, Japan \\ Department of Physics, Graduate School of Science, Kyoto University, \\ Kitashirakawa, Sakyo-ku, Kyoto 606-8502, Japan
}

(Received 3 June 2009 / Accepted 16 July 2009)

\begin{abstract}
In order to discuss the feasibility of compact neutron sources for scientific, medical and industrial applications, the yield of laser-produced neutrons is scaled by the laser energy. The laser energy scaling law of the neutron yield is derived from the laser intensity scaling law for the energy and the number of laser produced ions. High-energy ions are generated by Coulomb explosion of clusters through intense femtosecond laser-cluster interactions. The reactions of $\mathrm{D}(\mathrm{D}, \mathrm{n}) \mathrm{He}$ generating high yield even by relatively low deuterium energy and $\mathrm{Li}(\mathrm{p}, \mathrm{n}) \mathrm{Be}$ generating relatively low energy neutrons are discussed. The neutron yield of $D(D, n) H e$ determines the potential for using compact neutron sources with the aid of modern laser technology. In addition, $\mathrm{p}(\mathrm{Li}, \mathrm{n}) \mathrm{Be}$ shows much higher yield than $\mathrm{Li}(\mathrm{p}, \mathrm{n}) \mathrm{Be}$ with the assumption of Coulomb explosion of a cluster with a diameter of $500 \mathrm{~nm}$.

(c) 2009 The Japan Society of Plasma Science and Nuclear Fusion Research
\end{abstract}

Keywords: intense femtosecond laser, cluster, Coulomb explosion, neutron

DOI: $10.1585 /$ pfr.4.041

\section{Introduction}

When a laser pulse is focused onto the surface of certain materials, a high-temperature micro-plasma is produced, which is associated with the emission of different types of high-energy radiations (electrons, X-ray, ions, and photons). Although the study of laser plasmas was commenced immediately after the invention of lasers, research on radiation sources from a practical viewpoint has not been conducted as actively for the following reasons. For instance, the maximum ion energy $E_{\text {ion }}[\mathrm{MeV}]$ can be experimentally scaled as $E_{\text {ion }}=0.8 I_{\mathrm{L}} \lambda_{\mathrm{L}}^{2}\left(I_{\mathrm{L}} \leq 1\right)$ and $E_{\text {ion }}=0.8\left(I_{\mathrm{L}} \lambda_{\mathrm{L}}^{2}\right)^{0.25}\left(I_{\mathrm{L}} \geq 1\right)$, where $I_{\mathrm{L}}$ and $\lambda$ are the laser intensity in units of $10^{15} \mathrm{~W} / \mathrm{cm}^{2}$ and the laser wavelength in $\mu \mathrm{m}$, respectively [1]. (Note that this scale is comprehensively obtained from the experiments with nano second laser pulses and any planer and spherical targets.) In order to obtain ions with energy of several $100 \mathrm{keV}$, the laser intensity should be higher than $10^{14} \mathrm{~W} \mu \mathrm{m}^{2} / \mathrm{cm}^{2}$, and such high intensities were realized only at large-scale facilities for laser nuclear research. Therefore, laser-produced ion sources have not been considered superior to conventional accelerator-based sources from the point of view of facility size.

The remarkable progress of high-power lasers with respect to ultra-short pulse laser technology and chirped pulse amplification concepts in recent years has made it possible to obtain ultra high optical intensity even with tabletop-sized lasers [2]. As a result, different types of

author'se-mail: sakabe@laser.kuicr.kyoto-u.ac.jp laser-produced radiation have started to attract attention. In fact, in the past ten years, several types of nuclear reactions, such as the production of neutrons, RI, and positrons, have been demonstrated [3], and it can be said that laserproduced radiations will be a research subject for the development of next-generation radiation sources.

Techniques such as neutron scattering (diffraction), neutron radiography, prompt gamma ray analysis, and neutron capture therapy are available due to the fact that neutrons are characterized by strong ability for penetration of matter, high discrimination capability with respect to light elements, spin magnetic scattering, and so on. It is expected that these techniques can be applied in the scientific, medical, and industrial fields. However, in the current situation, conventional neutron source facilities, which are equipped with nuclear fission reactors or high-energy accelerators, are too large and expensive to be considered for mass production, and the number of available facilities of this kind is limited. Furthermore, researchers themselves face obstacles when using such facilities since they cannot perform preliminary experiments such as X-ray diffraction analysis in a laboratory with small-scale equipment before moving to large-scale light radiation facilities. The increasing dissemination of neutron use requires compact neutron sources.

In the future, with regard to laser-produced neutron sources, laser nuclear fusion reactors and laser accelerators will ultimately replace fission reactors and conventional accelerators, respectively, although this, however, is un- 
likely to result in decreasing the physical dimensions of such facilities. From the viewpoint of dissemination of neutron science, it is important to discuss the feasibility of compact neutron sources using ions generated by laserplasma interactions. Generally required neutron number is more than $10^{11}, 10^{12}$, and $10^{13}$ neutrons/s for scientific, medical and industrial applications, respectively.

Since Ditmire et al. first demonstrated the generation of neurons by irradiating intense laser pulses onto deuterium clusters [4], many groups have been studying laser-cluster interactions [5]. The authors were the first to provide experimental proof of the fact that cluster plasmas irradiated by intense femtosecond laser pulses do not expand, unlike micro-plasma spheres, but instead explode as a result of repulsive Coulomb forces after the instantaneous expulsion of electrons from the cluster [6]. Furthermore, the authors have derived the laser intensity scaling law for the energy of the ions emitted from Coulomb-exploding clusters. In this paper, based on the above results, we derive the laser energy scaling law for the yield of neutrons generated by ions produced in laser-cluster (nanoparticle) interactions, and discuss the feasibility of compact neutron sources.

\section{Generation of High-Energy Ions by Laser Cluster Interactions}

The generation of high-energy ions by interactions between intense femtosecond lasers and plasma can be realized via two mechanisms. One mechanism is based on acceleration in an electrostatic field induced by highenergy electrons driven by a ponderomotive force in overdense plasmas in thin foils [7,8], and the other involves Coulomb explosion in molecular gases, such as clusters or nano particles [6]. Here, we discuss the latter mechanism. When a cluster is irradiated with an intense femtosecond laser pulse, the following two phenomena can be considered. One phenomenon concerns the heating of the cluster until it is transformed into spherical plasma and the subsequent expansion of the created plasma as a result of plasma pressure. The other phenomenon is that electrons are instantaneously expelled from the cluster, and the ion cluster thus explodes under the influence of repulsive Coulomb forces. Although it cannot be directly observed, the type of phenomenon which occurs in the cluster can be determined from the energy distribution of the ions emitted from the cluster. The respective ion energy distributions resulting from the two phenomena are rather different: ions emitted from expanding spherical plasma generally exhibit a broad Boltzmann energy distribution $\mathrm{d} N / \mathrm{d} E \sim \exp \left(-E / k_{\mathrm{B}} T_{\mathrm{e}}\right)$, while ions generated by Coulomb explosion exhibit an energy distribution of $\mathrm{d} N / \mathrm{d} E \sim E^{1 / 2}$ with a finite maximum energy $E_{\max }$, as shown below. Thus, the Coulomb explosion produces a much greater number of high-energy ions.

The model for spherical Coulomb explosion is de- scribed in [6] and [9], and is briefly explained here. We consider a spherical cluster with a uniform density $n$ and a radius $R$ and assume equal electron and ion charge densities (average charge of ions $\bar{Z}$ ), where initially both components have zero temperature and are at rest. After the instantaneous expulsion of electrons, the ions (with a charge $Z$ ) accelerated by the Coulomb repulsive force have the maximum energy of

$$
\begin{aligned}
E_{\max } & =\frac{4}{3} \pi Z \bar{Z} e^{2} n R^{2} \\
& \cong 300 Z \bar{Z}\left(\frac{n}{5 \times 10^{22} \mathrm{~cm}^{-3}}\right)\left(\frac{R}{1 \mu \mathrm{m}}\right)^{2}[\mathrm{MeV}]
\end{aligned}
$$

and exhibit the following energy distribution:

$$
\frac{\mathrm{d} N}{\mathrm{~d} E_{\mathrm{i}}}=\frac{3}{4 Z \bar{Z} e^{2}} \sqrt{\frac{3 E_{\mathrm{i}}}{\pi n}} .
$$

Figure 1 (a) shows a schematization of the energy distribution of ions emitted from clusters. The ions with the maximum energy are emitted from the surface of the clusters, while the ions with lower energy originate from the inner regions of the clusters beyond its surface. Typical experimental results for energy distributions of ions emitted from hydrogen clusters are shown in Fig. 1 (b) [6]. From these experimental results, the authors have obtained experimental proof that clusters irradiated with intense femtosecond laser pulses do not exhibit thermal expansion but Coulomb explosion:

(1) The energy distribution is of the type $\mathrm{d} N / \mathrm{d} E \propto \sqrt{E}$ $(\mathrm{d} N / \mathrm{d} E \propto \exp (-E)$ in the case of thermal planer expansion) (Fig. 1 (b)) (see Fig. 1 in the first of [6], Fig. 3 in the second of [6]),

(2) For constant laser intensity, larger clusters emit ions with higher energy (ions with lower energy are emitted from larger clusters in the case of thermal expansion) (see Fig. 2 in the first of [6], Fig. 2 in the second of [6]),

(3) The ion energy for a constant cluster size does not increase even when increasing the intensity of the pulses (higher energy at higher intensity in the case of thermal expansion) (see Fig. 4 in the second of [6]).

Here, it should be noted that the energy distribution in Fig. 1 (b) is not as sharply cut at the maximum energy as that of Fig. 1 (a), which is due to the distribution of clusters in terms of size as discussed in [6]. In the following discussion, nano particles with well-controlled size are used, and therefore the energy distribution such as that shown in Fig. 1 (a) can be assumed.

\section{Laser Intensity Scaling Law for the Energy of Ions Emitted from a Coulomb-Exploded Cluster}

As discussed in Sec. 2, the ion energy is determined only by the cluster size, and a dependence on the laser in- 


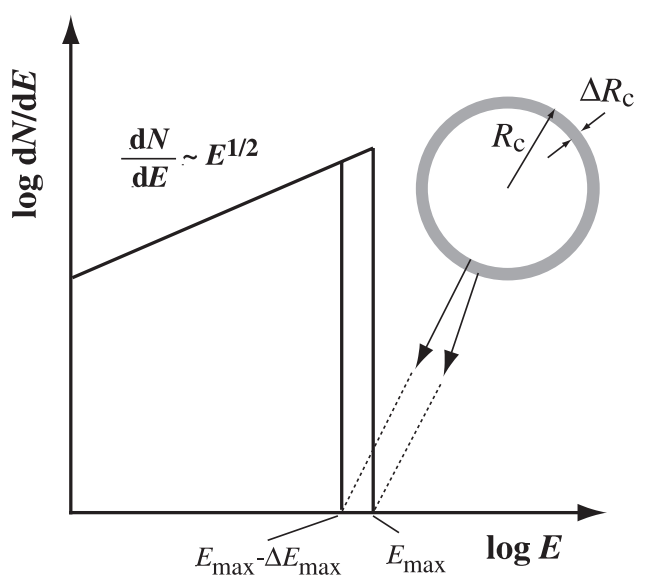

(a)

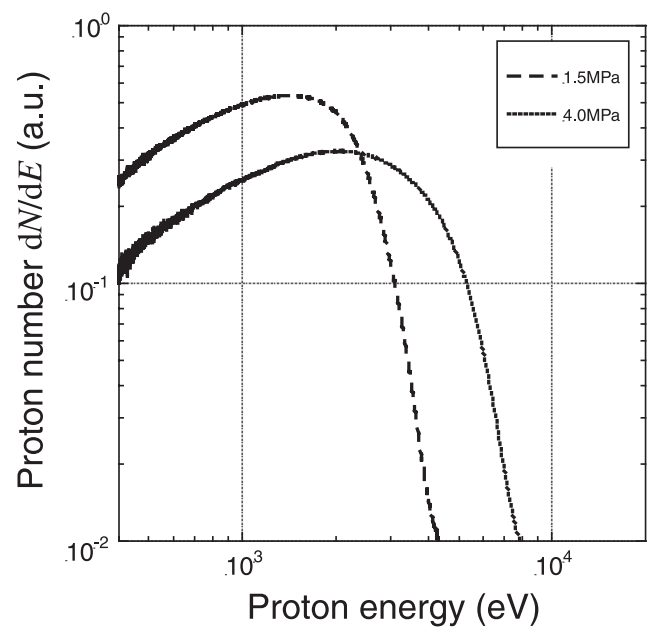

(b)

Fig. 1 (a) Energy distribution of ions emitted from a cluster, as obtained by using a spherical ion cluster model, (b) energy distribution of ions emitted from clusters irradiated with $100 \mathrm{fs}$ pulses with intensity of $1.6 \times 10^{16} \mathrm{~W} / \mathrm{cm}^{2}$ for different levels of the back pressure for generating clusters (higher pressure produces larger clusters) [6].

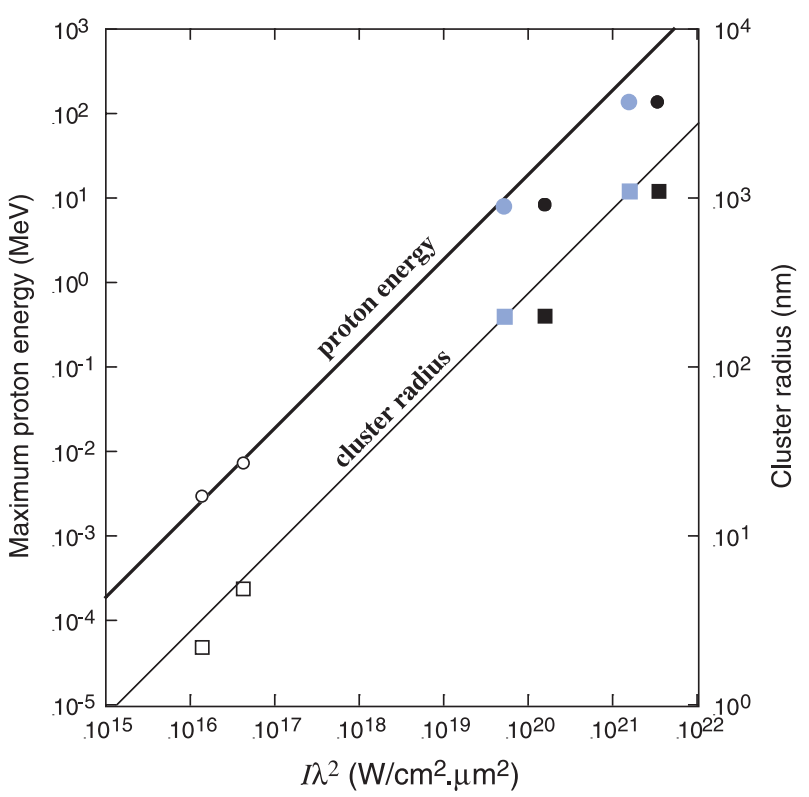

Laser intensity $x$ square of laser wavelength

Fig. 2 Laser intensity scaling laws for the radius of the cluster, in which electrons can be expelled by the laser pulse, and for the maximum energy of ions emitted from the cluster. Open symbols denote the experimental results presented in [6] and closed ones denote the results of the PIC simulations presented in [9]. Based on the assumption that the intensities for the PIC simulations are too high for the corresponding clusters given by the present scaling law since the cluster size is not optimized in these simulations, the shaded symbols are shifted toward the laser intensity in such a way that the closed ones lie on the thin line. These are revealed by gray closed symbols.

tensity appears in the process of electron expulsion. From the model mentioned above, the laser intensity required to expel electrons from the cluster is also reduced as fol- lows $[6,9]$

$$
\begin{aligned}
a_{0} & =\left\{\frac{I_{\mathrm{L}}\left[\mathrm{W} / \mathrm{cm}^{2}\right]}{1.37 \times 10^{18}}\left(\lambda_{\mathrm{L}}[\mu \mathrm{m}]\right)^{2}\right\}^{1 / 2} \\
& >\left(\frac{8 \pi \bar{Z} e^{2} n}{3 m c^{2}}\right)^{1 / 2} \\
& \cong 34\left(\frac{\bar{Z} n}{5 \times 10^{22} \mathrm{~cm}^{-3}}\right)^{1 / 2}\left(\frac{R}{1 \mu \mathrm{m}}\right),
\end{aligned}
$$

where $a_{0}$ is the normalized vector potential amplitude and $I_{\mathrm{L}}$ is the laser intensity. Then, the size of a cluster from which electrons can be expelled and the maximum ion energy can be scaled by the laser intensity as follows.

$$
\frac{R}{\mathrm{~nm}}=25\left(\frac{\bar{Z} n}{5 \times 10^{22} \mathrm{~cm}^{-3}}\right)^{-1 / 2}\left[\frac{I_{\mathrm{L}}}{10^{18} \mathrm{~W} / \mathrm{cm}^{2}}\left(\frac{\lambda_{\mathrm{L}}}{\mu \mathrm{m}}\right)^{2}\right]^{1 / 2},
$$

$$
\frac{E_{\max }}{\mathrm{MeV}}=0.19 Z \frac{I_{\mathrm{L}}}{10^{18} \mathrm{~W} / \mathrm{cm}^{2}}\left(\frac{\lambda_{\mathrm{L}}}{\mu \mathrm{m}}\right)^{2} .
$$

Figure 2 shows the dependence of the cluster radius and the maximum ion energy on the intensity of the laser for hydrogen gas $(\bar{Z}=Z=1)$. The adequacy of this model is verified on the basis of the satisfactory agreement with the experimental results obtained by the authors and the PIC simulation results presented in [9].

\section{Neutron Generation by Laser- Cluster Interactions}

The first demonstration of neutron generation resulting from the interaction between laser and clusters was presented by Ditmire et al., where they used deuterium 


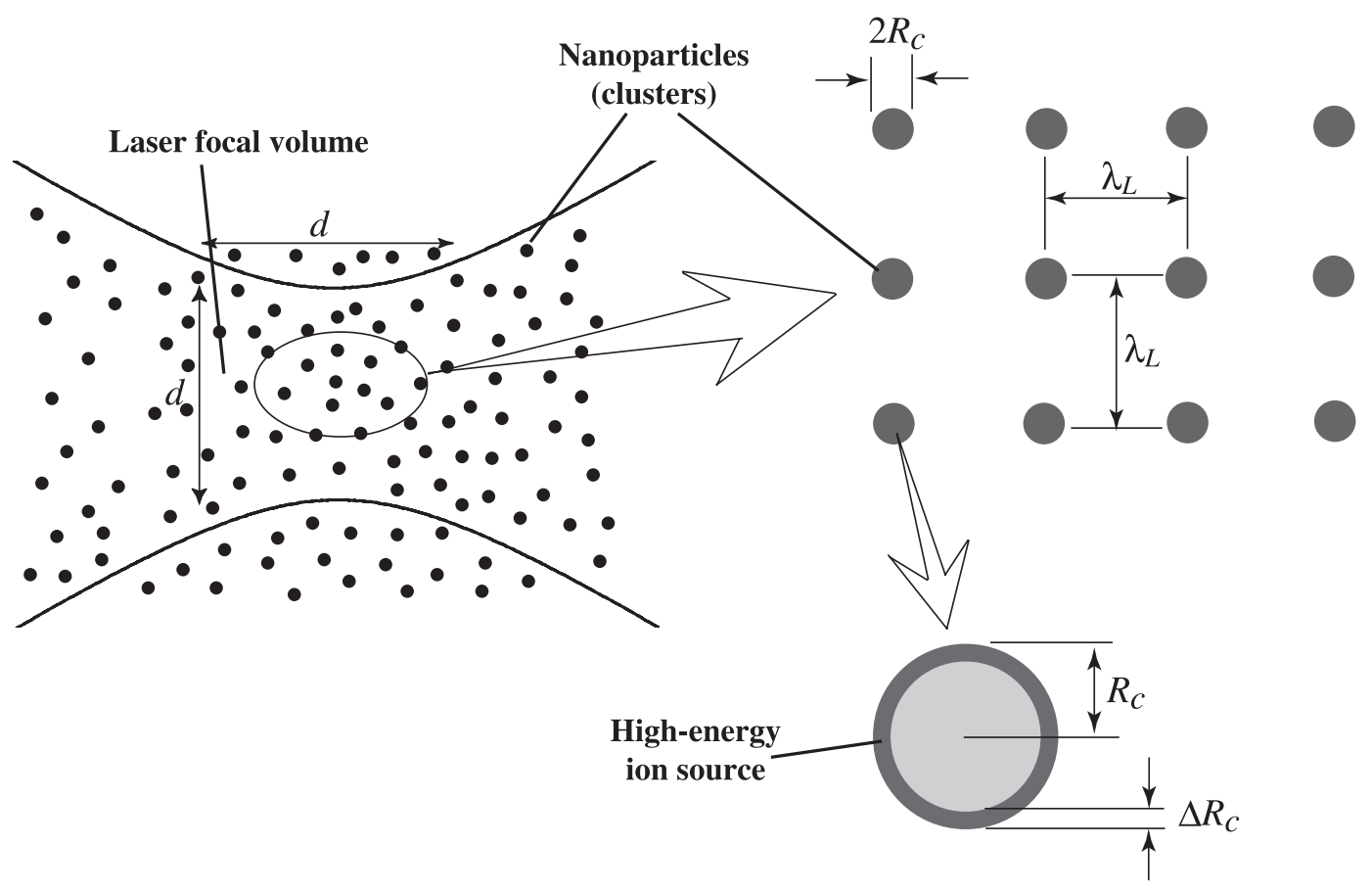

Fig. 3 The configuration of the clusters in the focal volume of the laser. The clusters are displaced with the interspace of the laser wavelength to neighbor clusters, therefore the coupling efficiency of a laser pulse to the cluster gas is assumed to be 1 .

clusters. Most likely, the deuterium ions emitted by the Coulomb explosion react with surrounding deuterium gas. In the present discussion, the reactive ions, which are generated by the Coulomb explosion of the clusters located in the focal volume of the laser, and the target atoms (solid or gas) are set as the Li walls or the surrounding hydrogen gas.

Here, we consider the following two reactions: (1) D $+\mathrm{D} \longrightarrow{ }^{3} \mathrm{He}+n(2.45 \mathrm{MeV})$ and $(2) p+{ }^{7} \mathrm{Li} \longrightarrow{ }^{7} \mathrm{Be}+$ $n-1.6 \mathrm{MeV}$. Although reaction (1) can be induced even by ions with relatively low energy $(>100 \mathrm{keV})$, the neutron energy is so high that it is necessary to use a large moderator and a shield in order to cool down the neutrons to cold neutrons. For instance the elastic scattering cross sections of neutrons in hydrogen atoms are 41 barns for 1-10 MeV neutrons, and 12-8 barns for 100$300 \mathrm{keV}$ neutrons. The energy of neutrons emitted from $\mathrm{p}+\mathrm{Li}$ reactions with $2.4 \mathrm{MeV}$ protons is about $300 \mathrm{keV}$. Therefore, on the contrary, although high-energy ions $(>2 \mathrm{MeV})$ are necessary for (2), it is not necessary to use an immense moderator and a shield due to the low energy of the produced neutrons. Regarding the ion sources, here we focus on $\mathrm{p}(2.25 \mathrm{MeV}), \mathrm{D}(100 \mathrm{keV})$, and $\mathrm{D}(2 \mathrm{MeV})$, where $\mathrm{p}(2.25 \mathrm{MeV})$ and $\mathrm{D}(2 \mathrm{MeV})$ have the maximum crosssections for the reactions. For instance, in order to produce $\mathrm{p}(2.25 \mathrm{MeV})$, clusters with a diameter of $172 \mathrm{~nm}$ are necessary, as seen from Fig. 3. Here, we assume that the clusters are placed in such a way that the average interval between neighbors is equal to the laser wavelength, as shown in Fig. 3. The density of protons with energy $E_{\max }$

$$
\begin{aligned}
& \sim E_{\max }-\Delta E_{\max } \text { is } \\
& n_{\mathrm{p}}=n_{\mathrm{s}} \frac{4 \pi R_{\mathrm{c}}^{2} \Delta R_{\mathrm{c}}}{4 / 3 \pi R_{\mathrm{c}}^{3}}\left(\frac{R_{\mathrm{c}}}{\lambda_{\mathrm{L}}}\right)^{3}=n_{\mathrm{s}} \frac{3 \Delta E_{\max }}{2 E_{\max }}\left(\frac{R_{\mathrm{c}}}{\lambda_{\mathrm{L}}}\right)^{3},
\end{aligned}
$$

where $n_{\mathrm{s}}$ is the density of the cluster (solid density). Assuming that the ions are emitted from a cylindrical volume whose diameter and length are equal to the diameter $d$ of the focal spot of the laser, the neutron yield is given by

$$
\begin{aligned}
N_{\mathrm{n}} & =n_{\mathrm{p}} \pi\left(\frac{d}{2}\right)^{2} \mathrm{~d} n_{\mathrm{Li}} \int_{0}^{R(E)} \sigma(E) \mathrm{d} z \\
& =\frac{\pi}{4} \mathrm{~d}^{3} n_{\mathrm{p}} n_{\mathrm{Li}} \int_{0}^{R(E)} \sigma(E) \mathrm{d} z
\end{aligned}
$$

where $n_{\mathrm{Li}}$ is the density of target $\mathrm{Li}, \sigma$ is the cross-section of the nuclear reaction, $z$ is the average flight distance for protons in the target, and $R(E)$ is the stopping distance for ions in the target. For a laser pulse with a duration $\tau_{\mathrm{L}}$, intensity $I_{\mathrm{L}}$, and energy $E_{\mathrm{L}}\left(I_{\mathrm{L}}=\frac{P_{\mathrm{L}}}{\pi(d / 2)^{2}}=\frac{4 P_{\mathrm{L}}}{\pi d^{2}}=\frac{4 E_{\mathrm{L}}}{\pi d^{2} \tau_{\mathrm{L}}}\right)$, the diameter $d$ of the focal spot of the laser is given by the laser energy $E_{\mathrm{L}}$, and the neutron yield can be scaled by the laser energy as follows:

$$
\begin{aligned}
N_{\mathrm{n}} & =\frac{2}{\pi^{1 / 2}}\left(\frac{E_{\mathrm{L}}}{I_{\mathrm{L}} \tau_{\mathrm{L}}}\right)^{3 / 2} n_{\mathrm{p}} n_{\mathrm{Li}} \int_{0}^{R(E)} \sigma(E) \mathrm{d} z \\
& =\frac{2}{\pi^{1 / 2}} \frac{n_{\mathrm{p}} n_{\mathrm{Li}}}{\left(I_{\mathrm{L}} \tau_{\mathrm{L}}\right)^{3 / 2}} \int_{0}^{R(E)} \sigma(E) \mathrm{d} z \cdot E_{\mathrm{L}}^{3 / 2}
\end{aligned}
$$


Table 1 Parameters for optimized Coulomb explosion of clusters and neutron yields.

\begin{tabular}{lllll}
\hline Reaction & Li(p, n)Be & D(D, n)He & D (D, n)He & p(Li, n)Be \\
\hline \hline Collision energy (eV) & $2.25 \mathrm{M}$ & $100 \mathrm{k}$ & $2 \mathrm{M}$ & $2.25 \times 7 \mathrm{M}$ \\
Laser intensity (W/cm $\left.{ }^{2}\right)$ & $1.9 \times 10^{19}$ & $8.2 \times 10^{17}$ & $1.6 \times 10^{19}$ & $1.3 \times 10^{20}$ \\
Cluster size (nm) & 172 & 36 & 162 & 455 \\
Reaction cross-section (barn) & 0.5 & $15 \mathrm{~m}$ & $90 \mathrm{~m}$ & 0.5 \\
\hline
\end{tabular}

Neutron yield

$2.1 \times 10^{6} E_{\mathrm{L}}^{3 / 2} 3.4 \times 10^{5} E_{\mathrm{L}}^{3 / 2} 2.1 \times 10^{8} E_{\mathrm{L}}^{3 / 2} 2.2 \times 10^{9} E_{\mathrm{L}}^{3 / 2}$

\section{Laser Energy Scaling Law of Neu- tron Yield}

The cluster diameters and the laser intensities necessary for producing the ions $\mathrm{p}(2.25 \mathrm{MeV}), \mathrm{D}(100 \mathrm{keV})$, and $\mathrm{D}(2 \mathrm{MeV})$ are shown in Table 1 together with the calculated laser energy laws of the neutron yield. Here, we have assumed a pulse duration of $100 \mathrm{fs}$ and $\Delta E_{\max } / E_{\max }=0.1$, that is, only the region within $5 \%$ of the cluster radius as measured from the surface of the cluster contributes to the emission of high-energy ions. Figure 4 shows a comparison of the present scaling for the $\mathrm{D}(\mathrm{D}, \mathrm{n}) \mathrm{He}(\mathrm{D}=100 \mathrm{keV})$ with respect to the experimental results reported in the relevant literature $[10,11]$. These two cited sources state different laser energy scaling laws, both of which are different from the scaling laws presented in this paper. As mentioned above, the ion energy is determined by the cluster size rather than by the intensity of the laser. In these experiments, the cluster size and the laser intensity are not optimized for producing neutrons. The present scaling law is obtained with constant laser intensity in order to expel electrons from the cluster, whose size is optimized to yield adequate ion energy. Therefore, optimized scaling is obtained, and in this sense, this law provides a satisfactory interpretation of the experimental results.

For the reaction of $\mathrm{p}(\mathrm{Li}, \mathrm{n}) \mathrm{Be}$, the yield is different from that for $\mathrm{Li}(\mathrm{p}, \mathrm{n}) \mathrm{Be}$. Li clusters are Coulomb-exploded to emit $\mathrm{Li}$ ions with an energy of $2.25 \times 7 \mathrm{MeV}$, which react with the surrounding hydrogen gas. For this reaction, the cluster size and the laser intensity must be $455 \mathrm{~nm}$ and $1.3 \times 10^{20} \mathrm{~W} / \mathrm{cm}^{2}$, respectively, in which high neutron yield $N_{\mathrm{n}}=2.2 \times 10^{9} E_{\mathrm{L}}^{3 / 2}$ is obtained. It is demonstrated by PIC simulations that even such large clusters Coulombexplode [9], although interactions between lasers and multiple large clusters arranged at intervals equal to the laser wavelength have not yet been experimentally studied and thus remain a topic for future research.

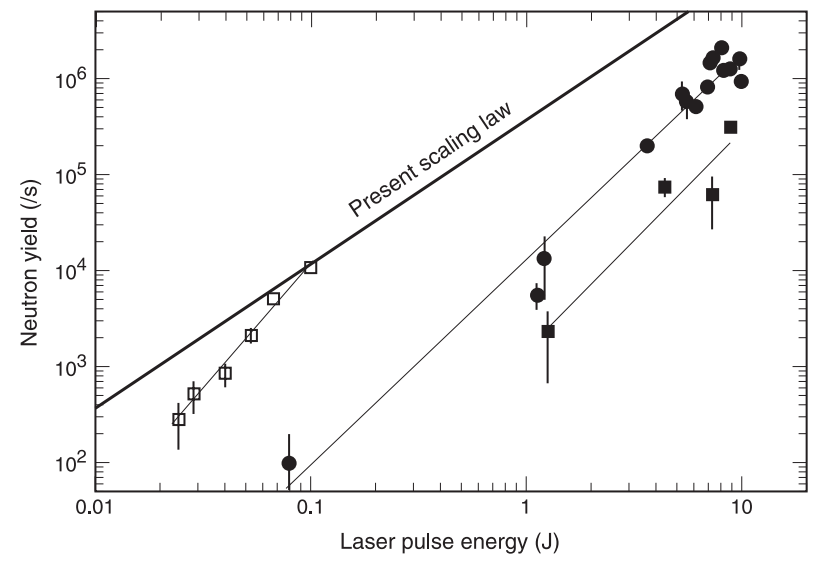

Fig. 4 Comparison between the present scaling law and the experimental results reported in [10] (open squares (35 fs laser pulses)) and [11] (circles (100 fs) and close squares (1 ps)) with regard to the dependence of the neutron yield on the laser energy. The present scaling is for the reactions of $\mathrm{D}(\mathrm{D}, n) \mathrm{He}(\mathrm{D}=100 \mathrm{keV})$.

\section{Feasibility of Neutron Sources}

In general, the neutron yield requirements for compact neutron sources are $>10^{11}$ neutrons/s for basic science, $>10^{12}$ neutrons/s for medical applications, and $>10^{13}$ neutrons/s for industry applications (such as semiconductor processing). Figure 5 shows the dependence of the number of neutrons on the laser energy for the reactions $\mathrm{Li}(\mathrm{p}, \mathrm{n}) \mathrm{Be}, \mathrm{D}(\mathrm{D}, \mathrm{n}) \mathrm{He}$, and $\mathrm{Li}(\mathrm{p}, \mathrm{n}) \mathrm{Be}$ with respect to the repetition rate of the laser pulses. For the reaction of $\mathrm{Li}(\mathrm{p}, \mathrm{n}) \mathrm{Be}$ (Fig. 5 (a)), a $1 \mathrm{~kJ} / 1 \mathrm{~Hz}$ laser is required in order to obtain $10^{11}$ neutrons/s. These characteristics are still difficult to obtain from the viewpoint of current laser technology. However, for the reaction of $\mathrm{D}(\mathrm{D}, \mathrm{n}) \mathrm{He}$ (Fig. 5 (b)), a $1 \mathrm{~J} / 1 \mathrm{kHz}$ laser can realize a neutron source yielding $10^{11}$ neutrons/s, and modern laser technology can produce such lasers. $100 \mathrm{~J} / 10 \mathrm{~Hz}$ lasers emitting $10^{12}$ neutrons/s are also 

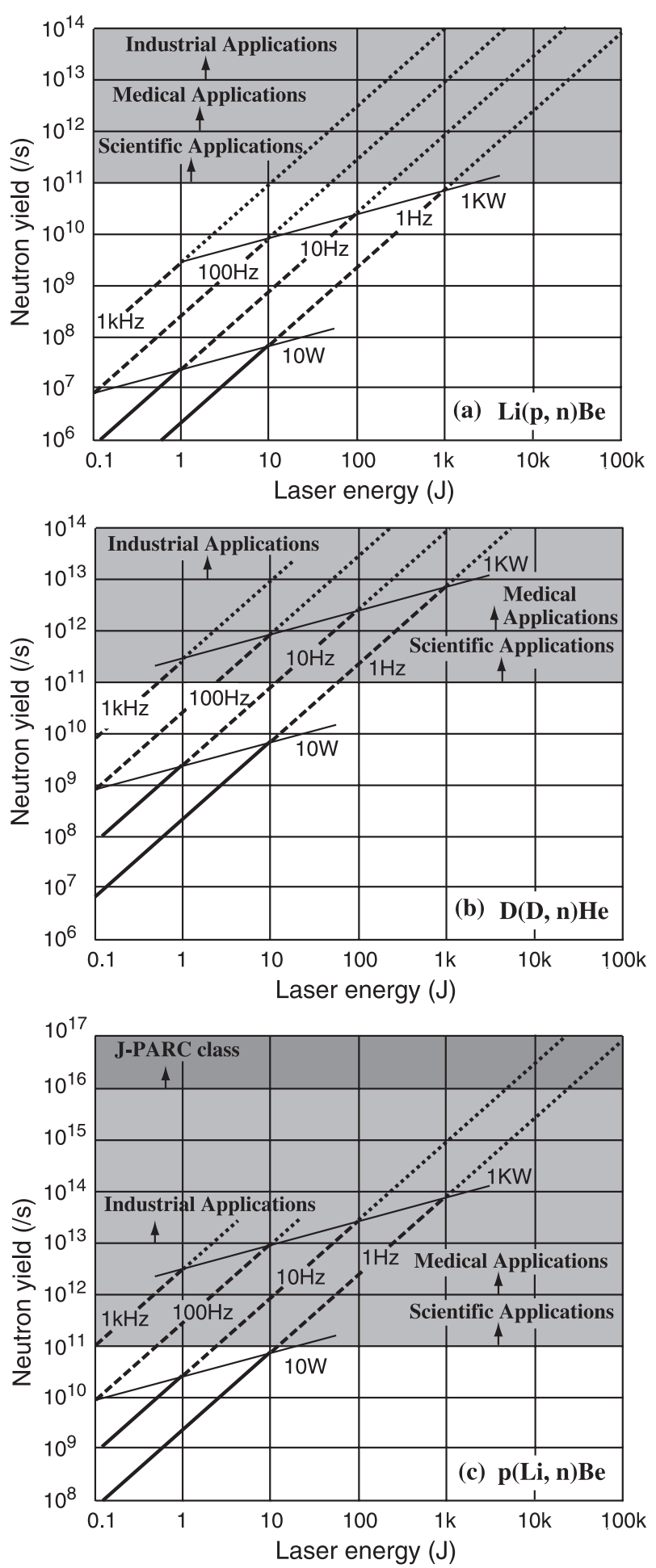

Fig. 5 Laser energy scaling laws for the yield of neutrons.

in the scope of current laser technology. For the reaction of p(Li,n)Be (Fig. 5 (c)), not only $10^{11}$ neutrons/s sources, but also $10^{13}$ neutrons/s ones can be realized, and additional benefits can be expected for this reaction. For example, the neutron energy can be reduced to several $100 \mathrm{keV}$, and therefore the size of the moderator can be reduced, while the moderator and the shield can be used as the target. If the $\mathrm{Li}$ ions emitted in isotropic manner from the focal point

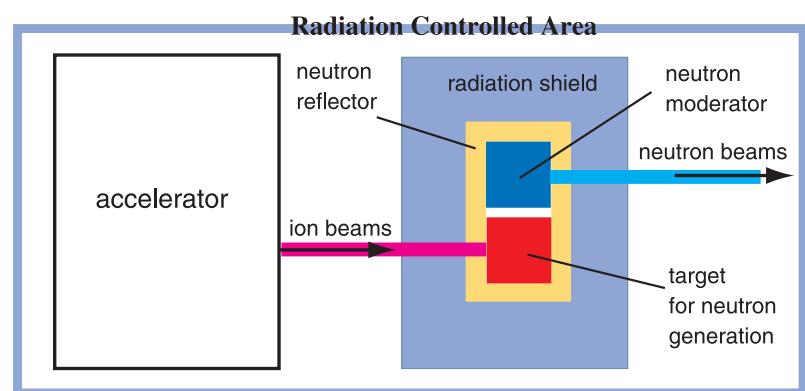

(a) Neutron source based on accelerators

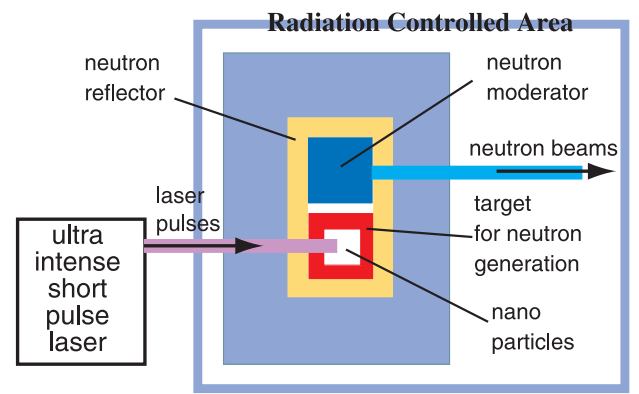

(b) Neutron source (the present concept)

Fig. 6 Schematic configurations of (a) conventional acceleratorbased neutron source and (b) the proposed neutron source generated by intense femtosecond laser -cluster interactions.

of the laser can be oriented in one or two directions towards the target / moderator by using electric or magnetic fields, neutrons can also be oriented due to the impingement of heavy Li ions with light protons. Therefore, great flexibility can be achieved in designing neutron source facilities with respect to moderators, shields, and transportation of neutron optics to the user sites. Finally, the laser system can be separated from the radiation-controlled area, as shown schematically in Fig. 6.

\section{Summary}

Neutron yields are estimated for reactions using ions emitted as a result of using intense femtosecond laser pulses to induce the Coulomb explosion of clusters. The laser energy scaling law for neutrons generated in the focal volume of the laser are derived on the basis of the laser intensity scaling laws for the size of the cluster, from which the laser can expel electrons, and for the maximum energy of ions emitted from the cluster. From the viewpoint of the number of emitted neutrons, compact neutron sources available for basic science, medical application, industrial processing, and so on can be realized with modern laser technology. Further development can lead to the stage of coordinating the process of designing relevant facilities, including targets / moderators and neutron transport lines in radiation shields, as well as laser systems outside radiation-controlled areas. 


\section{Acknowledgement}

This work was supported by a Grant-in-Aid for the Global COE Program "The Next Generation of Physics, Spun from Universality and Emergence" from the Ministry of Education, Culture, Sports, Science and Technology (MEXT) of Japan. The authors thank Toshiharu Kanaya for his comments as a user of a neutron facility, and Yoshihiko Shimizu for his comments on neutron facilities. We also thank the "Committee of Laser Neutron Sources and New Industry Creation” by IFE Forum, Institute of Laser Engineering, Osaka University, and The Graduate School for the Creation of New Photonic Industries for fruitful discussions.

[1] S. Sakabe, T. Mochizuki, T. Yabe, K. Mima and C. Yamanaka, Phys. Rev. A 26(4), 2159 (1982).

[2] G.A. Mourou, C.P.J. Garty, and M.D. Perry, Phys. Today 51, 22 (1988); M.D. Perry and G.A. Mourou, Science 264, 917 (1994); S. Sakabe, Rev. Laser Eng. 25, 855 (1997).

[3] C. Yamanaka and S. Sakabe, Rev. Laser Eng. 30, 185 (2002); S. Sakabe, T. Iida and A. Takahashi, J. Atomic Energy Soc. Japan, 43(10), 996 (2001).

[4] T. Ditmire, T. Donnely, A.M. Rubenchik, R.W. Falcone and M.D. Perry, Phys. Rev. A 53, 3379 (1996); T. Ditmire, J.W.G. Tisch, E. Springate, M.B. Mason, N. Hay, R.A. Smith, J. Marangos and M.H.R. Hutchinson, Nature (London) 386, 54 (1997); T. Ditmire, E. Springate, J.W.G.
Tisch, Y.L. Shao, M.B. Mason, N. Hay, J.P. Marangos, and M.H.R. Hutchinson, Phys. Rev. A 57, 369 (1998).

[5] References related to laser cluster interactions are listed in the Reference section of [6].

[6] S. Sakabe, S. Shimizu, M. Hashida, F. Sato, T. Tsuyukushi, K. Nishihara, S. Okihara, T. Kagawa,Y. Izawa, K. Imasaki and T. Iida, Phys. Rev. A 69(2), 23203 (2004); S. Sakabe, K. Shirai, M. Hashida, S. Shimizu and S. Masuno, Phys. Rev. A 74, 043205-1-5 (2006).

[7] A. Yogo, H. Daido H, A. Fukumi, Z. Li, K. Ogura, A. Sagisaka, A.S. Pirozhkov, S. Nakamura, Y. Iwashita, T. Shirai, A. Noda A, Y. Oishi, T. Nayuki T, T. Fujii, K. Nemoto, I.W. Choi, J.H. Sung, D.K. Ko DK, J. Lee, M. Kaneda and A. Itoh, Nucl. Phys. Plasmas 14(4), 043104 (2007).

[8] T. Zh. Esirkepov, S.V. Bulanov, K. Nishihara, T. Tajima, F.. Pegoraro, V.S. Khoroshkov, K. Mima, H. Daido, Y. Kato, Y. Kitagawa, K. Nagai and S. Sakabe, Phys. Rev. Lett. 89(17), 175003-1 (2002).

[9] K. Nishihara, H. Amitani, M. Murakami, S.V. Bulanov and T. Zh. Esirkepov, Nucl. Instrum. Methods Phys. Res. A 464, 98 (2001).

[10] J. Zweiback, T.E. Cowan, J.H. Hartley, R. Howell, K.B. Wharton, J.K. Crane, V.P. Yanovsky and G. Hays, Phys. Plasma 9(7), 3108 (2002).

[11] K.W. Madison, P.K. Patel, M. Allen, D. Price, R. Fitzpatrick and T. Ditmire, Phys. Rev. A 70(5), 053201 (2004). 